\title{
Gram-Schmidt Combined LLL Lattice-Reduction Aided Detection in MIMO Systems
}

\author{
Tadashi Fujino \\ Dept. Information and Communication Engineering and Informatics, \\ University of Electro-Communications, Tokyo, Japan \\ Correspondence: fujino@ice.uec.ac.jp, tad-fujino@mwb.biglobe.ne.jp \\ Manuscript communication: received 17 May 2011, accepted 17 June 2011
}

\begin{abstract}
This paper proposes an improved lattice-reduction aided (LRA) MMSE detection scheme, based on the GramSchmidt (GS) procedure. The proposed scheme reduces the column vectors of the MIMO channel matrix, by using the LLL algorithm followed by the GS procedure in order to transform the channel matrix into a new one which has mutually purely orthogonal column vectors. Compared to the conventional LRA MMSE detector, the proposed detector achieves a very good BER performance, almost equivalent to those using the ML detector in the $4 \times 4$ MIMO system at the cost of a slightly larger computational complexity.
\end{abstract}

Keywords- Gram-Schmidt orthogonalization, lattice-reduction, LLL algorithm, MIMO, ML, MMSE, signal estimation.

\section{INTRODUCTION}

Recently, lattice-reduction (LR) aided (LRA) detection has been receiving more attention since it might achieve a high channel capacity in multiple-input multiple-output (MIMO) systems. The LR transforms the column vectors of the MIMO channel matrix to become close to mutually orthogonal, followed by the estimation of the transmitted signals [1-8].

The most popular LR algorithm is the well-known LLL algorithm introduced by Lenstra, Lenstra, and Lovász [9]. Using this algorithm, the LRA detector gives a good signal estimation and, hence, a good biterror-rate (BER) performance, relatively close to that using the maximum likelihood (ML) detector [1-6]. However, the BER performance is not as good as that using the ML detector, since the column vectors of the reduced channel matrix are not mutually orthogonal.

In this paper, we propose an improved LRA minimum mean square error (MMSE) detection method. This is done by combing the LLL algorithm and the Gram-Schmidt (GS) orthogonalization procedure, aiming at achieving a BER performance almost equivalent to that using the ML detection at the cost of a slightly larger computational complexity. The feature of the LLL algorithm is to create the column vectors of the basis channel matrix nearly (but not purely) orthogonal. In addition, it yields the transform matrix with integer entries. This property facilitates to obtain a good estimate of the transmitted signal.

The motivation to bring in the Gram-Schmidt proce- dure is, from our intuition, that if we apply the GramSchmidt procedure to the LLL-reduced channel matrix, the column vectors of the reduced channel matrix should become more closely orthogonal and, hence, better estimate might be obtained. A disadvantage of this method is that the entries of the transform matrix are non-integer so that it would be very complicated in obtaining a good estimate of the signal. In this paper, we propose a novel method which overcomes this disadvantage.

It is well known that the LLL algorithm reduces the $p$-th column vector of the channel matrix by the $q$-th $(q<p)$ column vectors to create the reduced channel matrix and the transform matrix. We call this reduction the forward LR (F-LR). In addition, we reduce the $p$-th column vector by the $q$-th $(q>p)$ column vectors to create another reduced channel matrix and another transform matrix. We call this reduction the backward LR (B-LR) [5]. Furthermore, the proposed detector reduces the LLL-reduced column vectors using the GS procedure. Then the GS-reduced column vectors become mutually orthogonal and almost of equal length. As a result, the proposed detector improves the BER performance, which is almost equivalent to that using ML detection.

The remainder of this paper is organized as follows. Section 2 presents the system model and the conventional LRA detection. Section 3 presents the basic concept of the GS procedure combined with the LLL latticereduction. In Section 4, we propose a GS procedurebased LRA MMSE detection. Section 5 gives the computer simulation results and discussions. Finally, we summarize and conclude the paper in Section 6. 
Transmit antenna $1 \quad$ Receive antenna 1

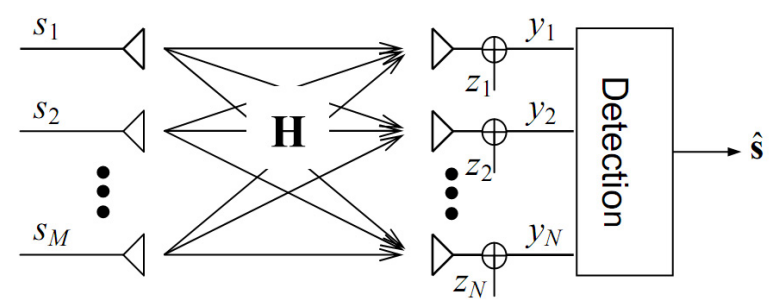

Transmit antenna $M \quad$ Receive antenna $N$

Figure 1. MIMO system model.

\section{System Model and Conventional LRA Detection}

\subsection{System Model}

Consider a MIMO system with $M$ transmit and $N$ receive antennas, $N \geq M$, as shown in Figure 1 . In the system, the signals are transmitted over a rich scattering flat fading channel. We assume that the receiver has a perfect knowledge of the channel state information (CSI). Let $\mathbf{H}=\left[\mathbf{h}_{1}, \ldots, \mathbf{h}_{M}\right]$ be the channel matrix of which the entry $h_{n, m}$ is the complex channel gain between the $m$-th transmit and the $n$-th receive antennas. The channel gains are assumed to be mutually uncorrelated complex Gaussian variables with zero mean and unity variance. Let $z_{n}$ be mutually uncorrelated additive white Gaussian noises at the $n$-th receivers. Each $z_{n}$ is assumed to be a complex Gaussian variable with zero mean and variance of $N_{0}$, where $N_{0}$ is the one-sided noise power spectral density. Let $\mathbf{s}=\left[s_{1}, \ldots, s_{M}\right]^{T}$, $\mathbf{y}=\left[y_{1}, \ldots, y_{N}\right]^{T}$, and $\mathbf{z}=\left[z_{1}, \ldots, z_{N}\right]^{T}$ be the transmit signal, the receive signal, and the additive noise vectors, respectively. Then we have

$$
\mathbf{y}=\mathbf{H s}+\mathbf{z}=\mathbf{h}_{1} s_{1}+\mathbf{h}_{2} s_{2}+\ldots+\mathbf{h}_{M} s_{M}+\mathbf{z}
$$

\subsection{LLL Algorithm}

The most popular LRA detection employs the LLL algorithm which is shown in Algorithm 1.

In this algorithm, using MMSE, the optimum value of $\delta$ in terms of achieving low BER performance is dependent on the MIMO size, the modulation order, and the signal-to-noise ratio (SNR), $E_{b} / N_{0}$. We will determine the value of $\delta$ in Section 5. The operator $\lceil x\rfloor$ denotes the rounding of $x$ in the real and the imaginary parts, separately. The algorithm transforms the channel matrix $\mathbf{H}$ to a reduced channel matrix $\mathbf{H}^{\prime}$ of which the column vectors are nearly pair-wise orthogonal, and a transform matrix $\mathbf{T}$ which is a unimodular matrix of determinant \pm 1 . Using $\mathbf{H}^{\prime}$ and $\mathbf{T}$, Equation (1) writes

$$
\mathbf{y}=\mathbf{H s}+\mathbf{z}=(\mathbf{H T})\left(\mathbf{T}^{-1} \mathbf{s}\right)+\mathbf{z} \equiv \mathbf{H}^{\prime} \mathbf{v}+\mathbf{z},
$$

where $\mathbf{H}^{\prime}=\mathbf{H T}$ and $\mathbf{v}=\mathbf{T}^{-1} \mathbf{s}$. Note that although steps (8)-(11) in Algorithm 1 are the GS procedure, they are used only for defining the swapping condition of the

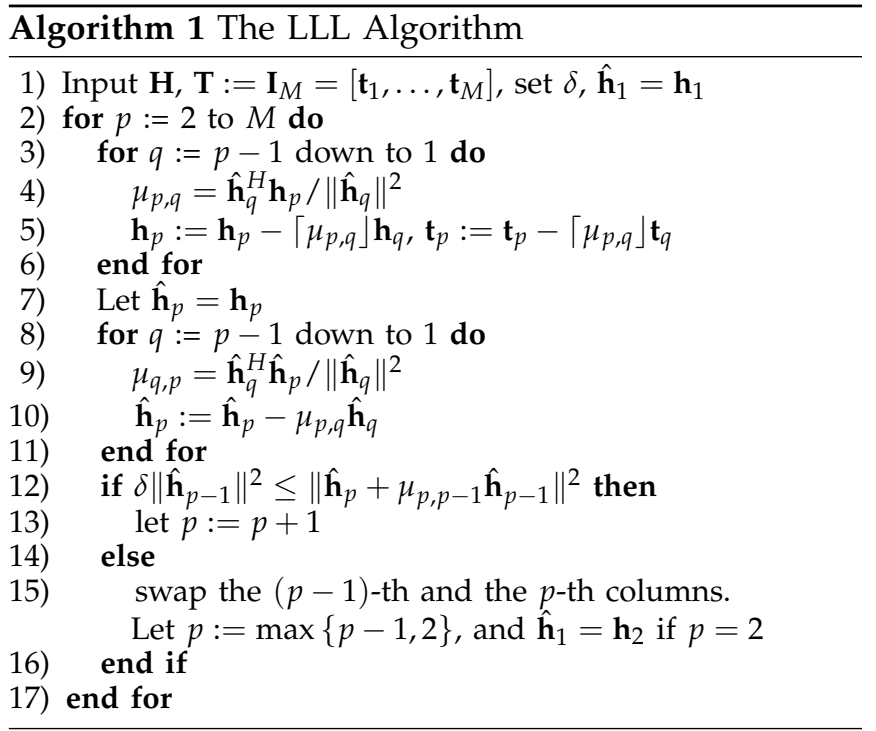

columns of $\mathbf{H}$ in steps (12)-(14). That is, the steps (8)(11) are applied regardless of the proposed GS-based LRA detection.

For the MMSE estimation, following Hassibi [10], define the extended receive signal vector, $\overline{\mathbf{y}}$, the extended channel matrix, $\overline{\mathbf{H}}$, and the extended additive noise vector, $\mathbf{z}$, respectively, as follows:

$$
\overline{\mathbf{y}}=\left[\begin{array}{c}
\mathbf{y} \\
\mathbf{0}_{M}
\end{array}\right], \overline{\mathbf{H}}=\left[\begin{array}{c}
\mathbf{H} \\
\sqrt{\rho} \mathbf{I}_{M}
\end{array}\right], \overline{\mathbf{z}}=\left[\begin{array}{c}
\mathbf{z} \\
-\sqrt{\rho} \mathbf{s}
\end{array}\right],
$$

where $1 / \rho=E_{s} / N_{0}$ with $E_{s}=E\left[\|\mathbf{s}\|^{2}\right] / M, \mathbf{I}_{M}$ is the $M \times M$ identity matrix, and $\mathbf{0}_{M}$ is the $M \times 1$ zero vector. Then, in step (1) of Algorithm 1, replace $\mathbf{H}$ with $\overline{\mathbf{H}}$. Next, the LLL-reduced channel matrix $\overline{\mathbf{H}}^{\prime}$ and the transform matrix $\mathbf{T}$ are obtained from the column vectors $\overline{\mathbf{h}}_{p}$ of $\overline{\mathbf{H}}$. Hence, Equation (2) extends to

$$
\overline{\mathbf{y}}=\overline{\mathbf{H}} \mathbf{s}+\overline{\mathbf{z}}=(\overline{\mathbf{H}} \mathbf{T})\left(\mathbf{T}^{-1} \mathbf{s}\right)+\overline{\mathbf{z}} \equiv \overline{\mathbf{H}}^{\prime} \mathbf{v}+\overline{\mathbf{z}},
$$

where $\overline{\mathbf{H}}=\overline{\mathbf{H}}^{\prime} \mathbf{T}$ and $\mathbf{v}=\mathbf{T}^{-1} \mathbf{s}$. Note that $\mathbf{T}$ and $\mathbf{v}$ in (4) are different from those in (2), respectively.

\subsection{Estimation of Transmitted Signal}

For MIMO detection, MMSE estimation is commonly used. In [10], Hassibi proposed an MMSE detector which is similar to the zero-forcing (ZF) detector with introduction of $\overline{\mathbf{y}}$ and $\overline{\mathbf{H}}$ in (3). Then, the MMSE estimation can be expressed by

$$
\tilde{\mathbf{s}}=\overline{\mathbf{H}}^{\dagger} \overline{\mathbf{y}}=\left(\mathbf{H}^{H} \mathbf{H}+\rho \mathbf{I}_{M}\right)^{-1} \mathbf{H}^{H} \mathbf{y},
$$

where $\overline{\mathbf{H}}^{\dagger}$ denotes the pseudo-inverse of $\overline{\mathbf{H}}$. Then, $\tilde{\mathbf{s}}$ is transformed to $\tilde{\mathbf{v}}=\mathbf{T}^{-1} \tilde{\mathbf{s}}$ in the $\mathbf{v}$-domain. In the case that the entries of $\mathbf{s}$ are of the commonly used quadrature amplitude modulation (QAM) mapping, proper shifting and scaling of $\tilde{\mathbf{s}}$ are necessary before determining $\tilde{\mathbf{v}}$. Detailed explanations on the shifting and scaling operations are given in [7]. Denote these operations in a combined form as $\tilde{\mathbf{s}}:=S[\tilde{\mathbf{s}}]$. After that, the entries of $\tilde{\mathbf{v}}$ are rounded as $\hat{\mathbf{v}}=\lceil\tilde{\mathbf{v}}]$. Next, $\hat{\mathbf{v}}$ is transformed to $\hat{\mathbf{s}}=\mathbf{T} \hat{\mathbf{v}}$ in the s-domain. Then, $\hat{\mathbf{s}}$ is shifted back and scaled back in the above case. 


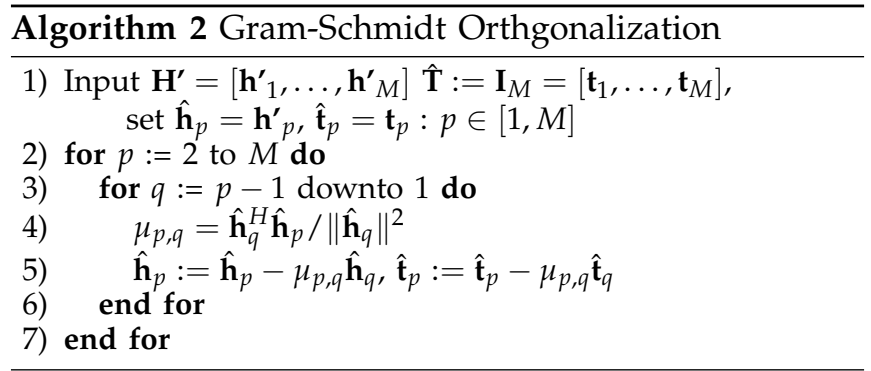

This is denoted by $\hat{\mathbf{s}}:=S^{-1}[\hat{\mathbf{s}}]$. At this stage, entries $\hat{s}_{m}, m \in[1, M]$, of $\hat{\mathbf{s}}$ are chosen to the nearest symbol constellation point if they are lying outside the symbol constellation. This operation is denoted by $\hat{\mathbf{s}}:=C[\hat{\mathbf{s}}]$.

\section{Basic Concept of GRAM-SChMidT Procedure Based MMSE Detection}

In this section, the basic concept of the proposed GS procedure based LRA MMSE detection is briefly explained. The LLL algorithm transforms the extended channel matrix $\overline{\mathbf{H}}$ to the reduced channel matrix $\overline{\mathbf{H}}^{\prime}$, of which the column vectors are nearly (but not purely) orthogonal to one another. The algorithm also makes the reduced column vectors almost of equal length. If the reduced column vectors were purely orthogonal to one another, no interference between the transmitted signals should occur. Hence, the BER performance should be equivalent to that using the ML detection. Unfortunately, the LLL algorithm does not make the column vectors of the reduced channel matrix $\overline{\mathbf{H}}^{\prime}$ mutually orthogonal. Henceforth, we will make them mutually orthogonal using the GS procedure, as described in Algorithm 2. This algorithm weakly reduces the column vectors of the LLL-reduced channel matrix $\overline{\mathbf{H}}^{\prime}$, forming the GS-reduced channel matrix $\hat{\mathbf{H}}$ and the transform matrix $\hat{\mathbf{T}}$. The column vectors of $\hat{\mathbf{H}}$ are mutually orthogonal. It should be noted that this algorithm is computationally simple since it weakly reduces the column vectors of $\mathbf{H}^{\prime}$.

For MMSE, the column vectors of $\overline{\mathbf{H}}^{\prime}$ are weakly reduced using this GS algorithm to create the GSreduced channel matrix $\hat{\mathbf{H}}$ and the transform matrix $\hat{\mathbf{T}}$. Then, (4) can be extended as

$$
\begin{aligned}
\overline{\mathbf{y}} & =\overline{\mathbf{H}} \mathbf{s}+\overline{\mathbf{z}}=(\overline{\mathbf{H}} \mathbf{T})\left(\mathbf{T}^{-1} \mathbf{s}\right)+\overline{\mathbf{z}} \equiv \overline{\mathbf{H}}^{\prime} \mathbf{v}+\overline{\mathbf{z}} \\
& =\left(\overline{\mathbf{H}}^{\prime} \hat{\mathbf{T}}\right)\left(\hat{\mathbf{T}}^{-1} \mathbf{v}\right)+\overline{\mathbf{z}} \equiv \hat{\mathbf{H}} \mathbf{u}+\overline{\mathbf{z}},
\end{aligned}
$$

where $\hat{\mathbf{H}}=\overline{\mathbf{H}}^{\prime} \hat{\mathbf{T}}=\overline{\mathbf{H} T \hat{\mathbf{T}}}, \mathbf{s}=\mathbf{T} \mathbf{v}=\mathbf{T} \hat{\mathbf{T}} \mathbf{u}, \mathbf{v}=\hat{\mathbf{T}} \mathbf{u}$, and $\operatorname{det}\{\mathbf{T} \hat{\mathbf{T}}\}= \pm 1$. Note that $\hat{\mathbf{T}}$ is an upper triangular matrix with unity diagonal entries and non-integer offdiagonal entries.

A soft estimate of $\mathbf{u}$ is expressed as $\tilde{\mathbf{u}}=(\mathbf{T} \hat{\mathbf{T}})^{-1} \tilde{\mathbf{s}}$. Since the entries of $\mathbf{u}\left(=(\mathbf{T} \hat{\mathbf{T}})^{-1} \mathbf{s}\right)$ are not integers, the entries of $\tilde{\mathbf{u}}$ cannot be decided by quantization like the LRA detection. The most critical problem for the proposed detection is how to decide $\tilde{\mathbf{u}}$ in order to obtain an estimate $\hat{\mathbf{u}}$ in the $\mathbf{u}$-domain. This problem will be uniquely solved using a novel method in Section 4 .
Once $\tilde{\mathbf{u}}$ is decided to be $\hat{\mathbf{u}}$, the estimate of the transmitted signal will then be obtained as $\hat{\mathbf{s}}=\mathbf{T} \hat{\mathbf{T}} \hat{\mathbf{u}}$, then let $\hat{\mathbf{s}}:=C\left[S^{-1}[\hat{\mathbf{s}}]\right]$.

\section{Proposed LRA MMSE Detection BASed on Gram-Schmidt Procedure}

In this section, the detailed procedure of the proposed GS-based LRA MMSE detection is explained.

\subsection{Forward and Backward LR Using LLL Algorithm}

First we forward-reduce the column vectors of $\overline{\mathbf{H}}=$ $\left[\overline{\mathbf{h}}_{1}, \ldots, \overline{\mathbf{h}}_{M}\right]$ using Algorithm 1. Input

$$
\begin{gathered}
\mathbf{H}=\left[\mathbf{h}_{1}, \ldots, \mathbf{h}_{M}\right]:=\overline{\mathbf{H}}, \\
\mathbf{T}:=\mathbf{I}_{M}=\left[\mathbf{t}_{1}, \ldots, \mathbf{t}_{M}\right]
\end{gathered}
$$

into Algorithm 1. Then we obtain the LLL-reduced channel matrix and the transform matrix, respectively, as below:

$$
\begin{gathered}
\overline{\mathbf{H}}^{\prime(j=1)} \equiv\left[\overline{\mathbf{h}}_{1}^{\prime(j=1)}, \ldots, \overline{\mathbf{h}}^{\prime}{ }_{M}^{(j=1)}\right]:=\left[\mathbf{h}_{1}, \ldots, \mathbf{h}_{M}\right], \\
\mathbf{T}^{(j=1)} \equiv\left[\mathbf{t}_{1}^{(j=1)}, \ldots, \mathbf{t}_{M}^{(j=1)}\right]:=\left[\mathbf{t}_{1}, \ldots, \mathbf{t}_{M}\right] .
\end{gathered}
$$

Next we backward-reduce the column vectors of $\overline{\mathbf{H}}$ using Algorithm 1. Input

$$
\begin{aligned}
\mathbf{H}=\left[\mathbf{h}_{1}, \ldots, \mathbf{h}_{M}\right] & :=\left[\overline{\mathbf{h}}_{M}, \ldots, \overline{\mathbf{h}}_{1}\right], \\
\mathbf{T}=\left[\mathbf{t}_{1}, \ldots, \mathbf{t}_{M}\right] & :=\left[\mathbf{t}_{M}, \ldots, \mathbf{t}_{1}\right],
\end{aligned}
$$

into Algorithm 1. Then we obtain the LLL-reduced channel matrix and the transform matrix

$$
\begin{gathered}
\overline{\mathbf{H}}^{\prime(j=2)} \equiv\left[\overline{\mathbf{h}}_{1}^{\prime(j=2)}, \ldots, \overline{\mathbf{h}}_{M}^{\prime(j=2)}\right]:=\left[\mathbf{h}_{M}, \ldots, \mathbf{h}_{1}\right], \\
\mathbf{T}^{(j=2)} \equiv\left[\mathbf{t}_{1}^{(j=2)}, \ldots, \mathbf{t}_{M}^{(j=2)}\right]:=\left[\mathbf{t}_{M}, \ldots, \mathbf{t}_{1}\right] .
\end{gathered}
$$

Here the superscripts $(j=1)$ and $(j=2)$ denote the F-LR and the B-LR of the extended channel matrix $\overline{\mathbf{H}}$, respectively.

\subsection{Gram-Schmidt Orthogonalization}

We next create eight GS-reduced channel matrices $\hat{\mathbf{H}}$ and their corresponding transform matrices $\hat{\mathbf{T}}$ by inputting $\overline{\mathbf{H}}^{(j)}, j \in\{1,2\}$, into Algorithm 2.

Case $1(k=1)$ : Forward GS-reduction of $\overline{\boldsymbol{H}}^{\prime(j)}$ : We input

$$
\begin{gathered}
\mathbf{H}^{\prime}=\left[\mathbf{h}^{\prime}{ }_{1}, \ldots, \mathbf{h}_{M}^{\prime}\right]:=\left[\overline{\mathbf{h}}_{1}^{\prime(j)}, \ldots, \overline{\mathbf{h}}^{\prime}{ }_{M}^{(j)}\right]=\overline{\mathbf{H}}^{\prime(j)}, \\
\hat{\mathbf{T}}=\left[\mathbf{t}_{1}, \ldots, \mathbf{t}_{M}\right]:=\mathbf{I}_{M} .
\end{gathered}
$$

Then, we obtain the GS-reduced channel matrix and the transform matrix, respectively, as

$$
\begin{aligned}
\hat{\mathbf{H}}^{(j, k=1)} \equiv\left[\hat{\mathbf{h}}_{1}^{(j, k=1)}, \ldots, \hat{\mathbf{h}}_{M}^{(j, k=1)}\right]:=\left[\hat{\mathbf{h}}_{1}, \ldots, \hat{\mathbf{h}}_{M}\right], \\
\hat{\mathbf{T}}^{(j, k=1)} \equiv\left[\hat{\mathbf{t}}_{1}^{(j, k=1)}, \ldots, \hat{\mathbf{t}}_{M}^{(j, k=1)}\right]:=\left[\hat{\mathbf{t}}_{1}, \ldots, \hat{\mathbf{t}}_{M}\right] .
\end{aligned}
$$


Case $2(k=2)$ : Backward GS-reduction of $\overline{\boldsymbol{H}}^{r^{(j)}}$ : Input

$$
\begin{aligned}
\mathbf{H}^{\prime}=\left[\mathbf{h}^{\prime}{ }_{1}, \ldots, \mathbf{h}^{\prime}{ }_{M}\right] & :=\left[\overline{\mathbf{h}}^{\prime}{ }_{M}^{(j)}, \ldots, \overline{\mathbf{h}}_{1}^{\prime(j)}\right], \\
\hat{\mathbf{T}}=\left[\mathbf{t}_{1}, \ldots, \mathbf{t}_{M}\right] & :=\left[\mathbf{t}_{M}, \ldots, \mathbf{t}_{1}\right] .
\end{aligned}
$$

Then, obtain

$$
\begin{aligned}
\hat{\mathbf{H}}^{(j, k=2)} \equiv\left[\hat{\mathbf{h}}_{1}^{(j, k=2)}, \ldots, \hat{\mathbf{h}}_{M}^{(j, k=2)}\right] & :=\left[\hat{\mathbf{h}}_{M}, \ldots, \hat{\mathbf{h}}_{1}\right], \\
\hat{\mathbf{T}}^{(j, k=2)} \equiv\left[\hat{\mathbf{t}}_{1}^{(j, k=2)}, \ldots, \hat{\mathbf{t}}_{M}^{(j, k=2)}\right] & :=\left[\hat{\mathbf{t}}_{M}, \ldots, \hat{\mathbf{t}}_{1}\right] .
\end{aligned}
$$

Case $3(k=3)$ : Forward GS-reduction of the columnorder rearranged $\overline{\boldsymbol{H}}^{\prime(j)}$ : Input

$$
\begin{aligned}
\mathbf{H}^{\prime} & =\left[\mathbf{h}^{\prime}{ }_{1}, \ldots, \mathbf{h}^{\prime}{ }_{M}\right] \\
& :=\left[\overline{\mathbf{h}}_{M / 2+1^{\prime}}, \ldots, \overline{\mathbf{h}}_{M}^{\prime(j)}, \overline{\mathbf{h}}_{1}^{(j)}, \ldots, \overline{\mathbf{h}}_{M / 2}^{(j)}\right], \\
\hat{\mathbf{T}} & =\left[\mathbf{t}_{1}, \ldots, \mathbf{t}_{M}\right]:=\left[\mathbf{t}_{M / 2+1}, \ldots, \mathbf{t}_{M}, \mathbf{t}_{1}, \ldots, \mathbf{t}_{M / 2}\right] .
\end{aligned}
$$

Then, obtain

$$
\begin{aligned}
& \hat{\mathbf{H}}^{(j, k=3)} \equiv\left[\hat{\mathbf{h}}_{1}^{(j, k=3)}, \ldots, \hat{\mathbf{h}}_{M}^{(j, k=3)}\right] \\
&:=\left[\hat{\mathbf{h}}_{M / 2+1}, \ldots, \hat{\mathbf{h}}_{M}, \hat{\mathbf{h}}_{1}, \ldots, \hat{\mathbf{h}}_{M / 2}\right], \\
& \hat{\mathbf{T}}^{(j, k=3)} \equiv\left[\begin{array}{l}
\left.\hat{\mathbf{t}}_{1}^{(j, k=3)}, \ldots, \hat{\mathbf{t}}_{M}^{(j, k=3)}\right] \\
:=\left[\hat{\mathbf{t}}_{M / 2+1}, \ldots, \hat{\mathbf{t}}_{M}, \hat{\mathbf{t}}_{1}, \ldots, \hat{\mathbf{t}}_{M / 2}\right] .
\end{array}\right.
\end{aligned}
$$

Case $4(k=4)$ : Backward GS-reduction of the columnorder rearranged $\overline{\boldsymbol{H}}^{\prime(j)}$ : Input

$$
\begin{aligned}
& \mathbf{H}^{\prime}=\left[\mathbf{h}^{\prime}{ }_{1}, \ldots, \mathbf{h}^{\prime}{ }_{M}\right] \\
& :=\left[\overline{\mathbf{h}}_{M / 2}^{\prime(j)}, \ldots, \overline{\mathbf{h}}_{1}^{\prime(j)}, \overline{\mathbf{h}}_{M}^{\prime(j)}, \ldots, \overline{\mathbf{h}}_{M / 2+1}^{\prime(j)}\right], \\
& \hat{\mathbf{T}}=\left[\mathbf{t}_{1}, \ldots, \mathbf{t}_{M}\right] \\
& :=\left[\mathbf{t}_{M / 2}, \ldots, \mathbf{t}_{1}, \mathbf{t}_{M}, \ldots, \mathbf{t}_{M / 2+1}\right] .
\end{aligned}
$$

Then, obtain

$$
\begin{aligned}
& \hat{\mathbf{H}}^{(j, k=4)} \equiv\left[\hat{\mathbf{h}}_{1}^{(j, k=4)}, \ldots, \hat{\mathbf{h}}_{M}^{(j, k=4)}\right] \\
& :=\left[\hat{\mathbf{h}}_{M / 2}, \ldots, \hat{\mathbf{h}}_{1}, \hat{\mathbf{h}}_{M}, \ldots, \hat{\mathbf{h}}_{M / 2+1}\right], \\
& \hat{\mathbf{T}}^{(j, k=4)} \equiv\left[\hat{\mathbf{t}}_{1}^{(j, k=4)}, \ldots, \hat{\mathbf{t}}_{M}^{(j, k=4)}\right] \\
& :=\left[\hat{\mathbf{t}}_{M / 2}, \ldots, \hat{\mathbf{t}}_{1}, \hat{\mathbf{t}}_{M}, \ldots, \hat{\mathbf{t}}_{M / 2+1}\right] .
\end{aligned}
$$

In Cases 1 and 2, the superscripts $(k=1)$ and $(k=2)$ denote the forward and the backward GS-reductions of $\overline{\mathbf{H}}^{\prime(j)}$, respectively, and, in Cases 3 and $4,(k=3)$ and $(k=4)$ denote the forward and the backward GS-reductions of the column-order rearranged $\overline{\mathbf{H}}^{\prime}(j)$, respectively. Again, remark that Algorithm 2 is computationally simple since it weakly reduces the column vectors of $\mathbf{H}^{\prime}$.

Finally, the extended receive signal vector $\overline{\mathbf{y}}$ is obtained by

$$
\begin{aligned}
\overline{\mathbf{y}} & =\overline{\mathbf{H}} \mathbf{s}+\overline{\mathbf{z}}=\left(\overline{\mathbf{H}} \mathbf{T}^{(j)}\right)\left(\mathbf{T}^{(j)-1} \mathbf{s}\right)+\overline{\mathbf{z}} \equiv \overline{\mathbf{H}}^{{ }^{(j)}} \mathbf{v}^{(j)}+\overline{\mathbf{z}} \\
& =\left(\overline{\mathbf{H}}^{\prime(j)} \hat{\mathbf{T}}^{(j, k)}\right)\left(\hat{\mathbf{T}}^{(j, k)-1} \mathbf{v}^{(j)}\right)+\overline{\mathbf{z}} \\
& \equiv\left(\overline{\mathbf{H}}_{\sim} \hat{\mathbf{T}}^{(j, k)}\right)\left(\hat{\mathbf{T}}^{(j, k)-1} \mathbf{s}\right)+\overline{\mathbf{z}} \equiv \hat{\mathbf{H}}^{(j, k)} \mathbf{u}^{(j, k)}+\overline{\mathbf{z}}
\end{aligned}
$$

(a)

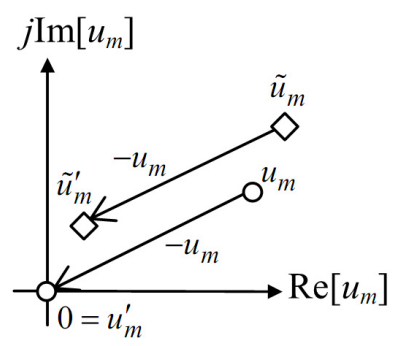

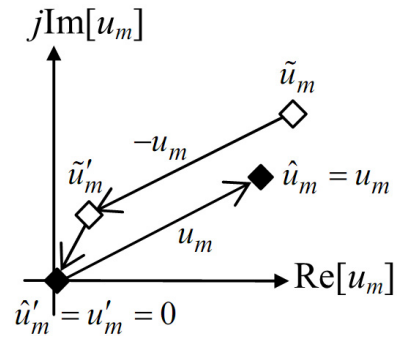

(b)
Figure 2. Decision flow of the soft estimate $\tilde{u}_{m}: \Delta u_{m}=-u_{m}$. (a)Shifting of $u_{m}$ and $\tilde{u}_{m} ;(\mathrm{b})-$ Quantization of $\tilde{u}_{m}^{\prime}$ and shifting back of $\tilde{u}_{m}^{\prime}$.

where $j \in\{1,2\}, k \in[1,4], \hat{\sim}^{(j, k)}=\mathbf{T}^{(j)} \hat{\mathbf{T}}^{(j, k)}, \hat{\mathbf{H}}^{(j, k)}=$ $\overline{\mathbf{H}}^{\prime} \hat{\mathbf{T}}^{(j, k)}=\overline{\mathbf{H}}_{\sim} \hat{\mathbf{T}}^{(j, k)}$, and

$$
\begin{gathered}
\mathbf{s}=\mathbf{T}^{(j)} \mathbf{v}^{(j)}=\hat{\mathbf{T}}^{(j, k)} \mathbf{u}^{(j, k)} \\
\mathbf{v}^{(j)}=\hat{\mathbf{T}}^{(j, k)} \mathbf{u}^{(j, k)}
\end{gathered}
$$

Note that the entries of $\hat{\mathbf{T}}^{(j, k)}$, of $\hat{\mathbf{T}}^{(j, k)}$ and of $\mathbf{u}^{(j, k)}$ are non-integers, and that $\operatorname{det}\{\underset{\sim}{\hat{\mathbf{T}}(j, k)}\}= \pm 1, \operatorname{det}\left\{\mathbf{T}^{(j)}\right\}=$ \pm 1 and $\operatorname{det}\left\{\hat{\mathbf{T}}^{(j, k)}\right\}= \pm 1$.

\subsection{Estimation of Signal Vector $\mathbf{u}$}

Although the receiver has no knowledge of the transmitted signal s, we at first assume that it should have knowledge of $\mathbf{s}$. Under this assumption, the transmitted signal $\mathbf{s}$ is first shifted and scaled as $\mathbf{s}:=S[\mathbf{s}]$. Then it is transformed to $\mathbf{u}^{(j, k)}$ as

$$
\mathbf{u}^{(j, k)}=\left[u_{1}^{(j, k)}, \ldots, u_{M}^{(j, k)}\right]^{T}=\hat{\mathbf{T}}^{(j, k)-1} \mathbf{s}
$$

where $j \in\{1,2\}$ and $k \in[1,4]$. Then we measure the distance between $\mathbf{u}^{(j, k)}$ and the origin $\mathbf{0}_{M}$ by

$$
\Delta \mathbf{u}^{(j, k)}=\mathbf{0}_{M}-\mathbf{u}^{(j, k)}=-\mathbf{u}^{(j, k)}=-{\underset{\sim}{\hat{\mathbf{T}}}}^{(j, k)-1} \mathbf{s}
$$

The $m$-th entry of $\Delta \mathbf{u}^{(j, k)}$ is expressed as $\Delta u_{m}^{(j, k)}=$ $-u_{m}^{(j, k)}$. After $\tilde{\mathbf{s}}$ in (5) is shifted and scaled as $\tilde{\mathbf{s}}:=S[\tilde{\mathbf{s}}]$, the soft estimate of $\mathbf{u}^{(j, k)}$ is derived from $\tilde{\mathbf{s}}$ as

$$
\tilde{\mathbf{u}}^{(j, k)}=\left[\tilde{u}_{1}^{(j, k)}, \ldots, \tilde{u}_{M}^{(j, k)}\right]^{T}=\hat{\sim}^{(j, k)-1} \tilde{\mathbf{s}}
$$

Now we have obtained the correct point $u_{m}^{(j, k)}$ in (10) and the soft estimate $\tilde{u}_{m}^{(j, k)}$ in (12). Since $u_{m}^{(j, k)}$ is not an integer, $\tilde{u}_{m}^{(j, k)}$ cannot be decided by quantization like the conventional LRA detector. In order to decide $\tilde{u}_{m}^{(j, k)}$, we shift both $u_{m}^{(j, k)}$ and $\tilde{u}_{m}^{(j, k)}$ by $\Delta u_{m}^{(j, k)}\left(=-u_{m}^{(j, k)}\right)$ such that $u_{m}^{(j, k)}$ should be shifted to the origin. Then the shifted $u_{m}^{(j, k)}$ and $\tilde{u}_{m}^{(j, k)}$ are expressed, respectively, as

$$
\begin{aligned}
& u_{m}^{\prime(j, k)}=u_{m}^{(j, k)}+\Delta u_{m}^{(j, k)}=u_{m}^{(j, k)}-u_{m}^{(j, k)}=0 \\
& \tilde{u}_{m}^{\prime(j, k)}=\tilde{u}_{m}^{(j, k)}+\Delta u_{m}^{(j, k)}=\tilde{u}_{m}^{(j, k)}-u_{m}^{(j, k)}
\end{aligned}
$$

Figure 2(a) illustrates the shifting of $u_{m}^{(j, k)}$ and $\tilde{u}_{m}^{(j, k)}$ by $\Delta u_{m}^{(j, k)}\left(=-u_{m}^{(j, k)}\right)$. In the figure, the superscript $(j, k)$ is omitted. 
Since ${u^{\prime}}_{m}^{(j, k)}$ is an integer (zero), $\tilde{u}_{m}^{(j, k)}$ can be rounded as

$$
{\hat{u}^{\prime}}_{m}^{(j, k)}=\left\lceil\tilde{u}_{m}^{(j, k)}\right\rfloor=\left\lceil\tilde{u}_{m}^{(j, k)}-u_{m}^{(j, k)}\right\rfloor
$$

After that, shift back ${\hat{u^{\prime}}}_{m}^{(j, k)}$ by $-\Delta u_{m}^{(j, k)}\left(=u_{m}^{(j, k)}\right)$ to create the estimate $\hat{u}_{m}^{(j, k)}$ as

$$
\hat{u}_{m}^{(j, k)}=\hat{u}_{m}^{(j, k)}-\Delta u_{m}^{(j, k)}=\left[\tilde{u}_{m}^{(j, k)}-u_{m}^{(j, k)}\right]+u_{m}^{(j, k)}
$$

Figure 2(b) illustrates an example of rounding of $\tilde{u}_{m}^{\prime(j, k)}$ to create $\hat{u}_{m}^{(j, k)}$. It also illustrates the shifting back of $\hat{u}_{m}^{(j, k)}$ by $-\Delta u_{m}^{(j, k)}\left(=u_{m}^{(j, k)}\right)$ to create the decided estimate $\hat{u}_{m}^{(j, k)}$.

Using (10), Equation (16) is expressed in vector form as

$$
\begin{aligned}
\hat{\mathbf{u}}^{(j, k)}= & \hat{\mathbf{u}}^{\prime(j, k)}-\Delta \hat{\mathbf{u}}^{(j, k)}= \\
& {\left[\tilde{\mathbf{u}}^{(j, k)}-{\underset{\sim}{\mathbf{T}}}^{(j, k)-1} \mathbf{s}\right]+{\underset{\sim}{\mathbf{T}}}^{(j, k)-1} \mathbf{s} }
\end{aligned}
$$

Here, it should be mentioned that pre-multiplication of $\hat{\mathbf{u}}^{(j, k)}$ in (17) by $\hat{\mathbf{T}}^{(j, k)}$ transforms $\hat{\mathbf{u}}^{(j, k)}$ to $\hat{\mathbf{v}}^{(j, k)}:=$ $\hat{\mathbf{T}}^{(j, k)} \hat{\mathbf{u}}^{(j, k)}$ in the v-domain of which the entries should be integers. Hence, using (17), we express $\hat{\mathbf{v}}^{(j, k)}$ as

$$
\begin{aligned}
\hat{\mathbf{v}}^{(j, k)}= & \left\lceil\hat{\mathbf{T}}^{(j, k)} \hat{\mathbf{u}}^{(j, k)}\right\rfloor= \\
& \left\lceil\hat{\mathbf{T}}^{(j, k)}\left\{\left\lceil\tilde{\mathbf{u}}^{(j, k)}-{\underset{\sim}{\mathbf{T}}}^{(j, k)-1} \mathbf{s}\right\rfloor+{\underset{\sim}{\mathbf{T}}}^{(j, k)-1} \mathbf{s}\right\}\right]
\end{aligned}
$$

Pre-multiplying $\hat{\mathbf{v}}^{(j, k)}$ in (18) by $\hat{\mathbf{T}}^{(j, k)-1}$, the estimate $\hat{\mathbf{v}}^{(j, k)}$ in (18) is transformed back to $\hat{\mathbf{u}}^{(j, k)}$ in the $\mathbf{u}$ domain as

$$
\begin{aligned}
& \hat{\mathbf{u}}^{(j, k)}=\hat{\mathbf{T}}^{(j, k)-1} \hat{\mathbf{v}}^{(j, k)}= \\
& \hat{\mathbf{T}}^{(j, k)-1}\left[\hat{\mathbf{T}}^{(j, k)}\left\{\left[\tilde{\mathbf{u}}^{(j, k)}-{\underset{\sim}{\mathbf{T}}}^{(j, k)-1} \mathbf{s}\right]+{\underset{\sim}{\mathbf{T}}}^{(j, k)-1} \mathbf{s}\right\}\right]
\end{aligned}
$$

Since the receiver practically has no knowledge of the transmitted signal $\mathbf{s}$, we here pre-estimate $\mathbf{s}$ in (19) using the conventional LRA MMSE detection. First, derive the soft estimate $\tilde{\mathbf{s}}$ in (5). Then let $\tilde{\mathbf{s}}:=S[\tilde{\mathbf{s}}]$. Next, transform $\tilde{\mathbf{s}}$ to $\tilde{\mathbf{v}}^{(j)}=\mathbf{T}^{(j)-1} \tilde{\mathbf{s}}$. Then, round the entries of $\tilde{\mathbf{v}}^{(j)}$ as $\hat{\mathbf{v}}^{(j)}=\left\lceil\tilde{\mathbf{v}}^{(j)}\right\rfloor$. Finally, transform $\hat{\mathbf{v}}^{(j)}$ to obtain

$$
\hat{\mathbf{s}}^{\prime(j)}=\mathbf{T}^{(j)} \hat{\mathbf{v}}^{(j)}
$$

Substitute $\hat{\mathbf{s}}^{\prime(j)}$ into $\mathbf{s}$ in (19) to revise $\hat{\mathbf{u}}^{(j, k)}$. Then, substitute $\hat{\mathbf{u}}^{(j, k)}$ into $\mathbf{u}^{(j, k)}$ in (8) to get the estimate $\hat{\mathbf{s}}^{(j, k)}$.

Note that the cross-correlation of $\mathbf{T}^{(j=1)}$ and $\mathbf{T}^{(j=2)}$ is weak as shown in [5]. Hence it is unlikely that both $\hat{\mathbf{s}}^{\prime}(j)$, $j \in\{1,2\}$, in (20) are in error at the same time. Similarly, the cross-correlation of ${\underset{\sim}{\mathbf{T}}}^{(j, k)}$ and $\hat{\mathbf{T}}^{\left(j^{\prime}, k^{\prime}\right)}$ is weak, where $j^{\prime} \neq j$ and $/$ or $k^{\prime} \neq k$. Hence it is unlikely that all $\hat{\mathbf{s}}^{(j, k)}(=$ $\left.\hat{\mathbf{T}}^{(j, k)} \hat{\mathbf{u}}^{(j, k)}\right), j \in\{1,2\}$ and $k \in[1,4]$, are in error at the same time. As a result, a good BER performance should be expected by selecting the most reliable estimate $\hat{\mathbf{s}}^{(j, k)}$.

\subsection{List of $\hat{u}$ and Estimation of $s$}

We here derive the estimate of the transmitted signal $\mathbf{s}$ using the proposed GS-based LRA MMSE detection. Replacing $\mathbf{s}$ in (19) by $\hat{\mathbf{s}}^{\prime(j)}$ in (20), we express the revised $\hat{\mathbf{u}}^{(j, k)}$ as $\hat{\mathbf{u}}^{(p=0, j, k)}$, which is first listed. Since $\tilde{\mathbf{u}}^{(j, k)} \approx \mathbf{u}^{(j, k)}$ in the high $E_{b} / N_{0}$ region, we further create $\hat{\mathbf{u}}^{(p, j, k)}, p \in[1, M]$, by replacing the $p$-th entry of $\hat{\mathbf{u}}^{(0, j, k)}$ by $\tilde{u}_{p}^{(j, k)}$ in (12), and add them to the list. By adding $\hat{\mathbf{u}}^{(p, j, k)}, p \in[1, M]$, to the list, a more reliable estimate of $\mathbf{s}$ is expected. Calculating $\hat{\mathbf{s}}^{(p, j, k)}=\hat{\mathbf{T}}_{\tilde{\mathbf{S}}}^{(j, k)} \hat{\mathbf{u}}^{(p, j, k)}$, $p \in[0, M]$, then letting $\hat{\mathbf{s}}^{(p, j, k)}:=S^{-1}\left[\tilde{\hat{\mathbf{s}}}^{\left(p_{j}, j, k\right)}\right]$ and $\hat{\mathbf{s}}^{(p, j, k)}:=C\left[\hat{\mathbf{s}}^{(p, j, k)}\right]$, select the most reliable signal among all $\hat{\mathbf{s}}^{(p, j, k)}$, where $p \in[0, M], j \in\{1,2\}$ and $k \in[1,4]$. The above procedure is summarized in Algorithm 3 , where the notations $S[\cdot]$ and $S^{-1}[\cdot]$ for $\hat{\mathbf{s}}^{(p, j, k)}$, $\hat{\mathbf{s}}^{[i, j, k]}, \hat{\mathbf{s}}^{\prime(j)}$ and $\hat{\mathbf{s}}^{\prime\{i\}}$ are omitted. In this algorithm, we obtain the estimate $\hat{\mathbf{s}}$ at step (49). We here call $\hat{\mathbf{s}}$ the GS-estimate. We also call this detection procedure the Gram-Schmidt combined forward and backward LRA (GSFEB-LRA) MMSE list detection. In order to achieve a more reliable GS-estimate of $\hat{\mathbf{s}}$, we replace $\hat{\mathbf{s}}^{\prime(i)}$ at step (9) by the updated estimate $\hat{\mathbf{s}}^{(p=M, j, k)}$ at step (21) for each $j$ and $k$ iteratively, where $i$ is the iteration number and $I$ is the number of iterations.

\section{Simulation Results and Discussions}

Computer simulations were carried out for QPSK and 16QAM in the $4 \times 4$ MIMO system to estimate the transmitted signals using the proposed GS-F\&B-LRA MMSE list detection without forward error correction. Each channel is assumed to be non-frequency selective slow-varying fading channel. The receiver is assumed to have perfect knowledge of the CSI. In the simulations, channel gains are generated using independent and identically distributed (i.i.d.) Gaussian random variables with zero mean and variance of $1 / 2$ for each dimension. Additive noise at each receive antenna is generated using i.i.d. Gaussian random variables with zero mean and variance of $N_{0} / 2$ for each dimension.

Before calculating the BERs for the proposed detection, we first determine the suitable values of $\delta$ in Algorithm 1 and the suitable number of iterations $I$ in Algorithm 3. After that, we analyze the BER performances and the computational complexity.

\subsection{Suitable Values for Factor $\delta$ and Number of Iterations $I$}

We here determine the suitable values of $\delta$ and the suitable number of $I$ for both the conventional and the proposed detections in the $4 \times 4 \mathrm{MIMO}$ system. We look for suitable value of $\delta$ that should achieve BER of $10^{-4}$ to $10^{-5}$ at the lowest $E_{b} / N_{0}$.

Figures 3(a) shows the characteristics of BERs versus $\delta$ with various values of $I$, for QPSK and 16QAM at $E_{b} / N_{0}=16 \mathrm{~dB}$ over the $4 \times 4$ MIMO channel. Figure 3(b) show the characteristics of the number of swapping times versus $\delta$ for 16QAM at $E_{b} / N_{0}=21 \mathrm{~dB}$.

Figures 4(a) and 4(b), respectively, show the BER characteristics versus the number of iterations $I$ for the proposed detection, for QPSK at $E_{b} / N_{0}=16 \mathrm{~dB}$ and 16QAM and at $E_{b} / N_{0}=21 \mathrm{~dB}$ over the $4 \times 4 \mathrm{MIMO}$ channel, respectively. 


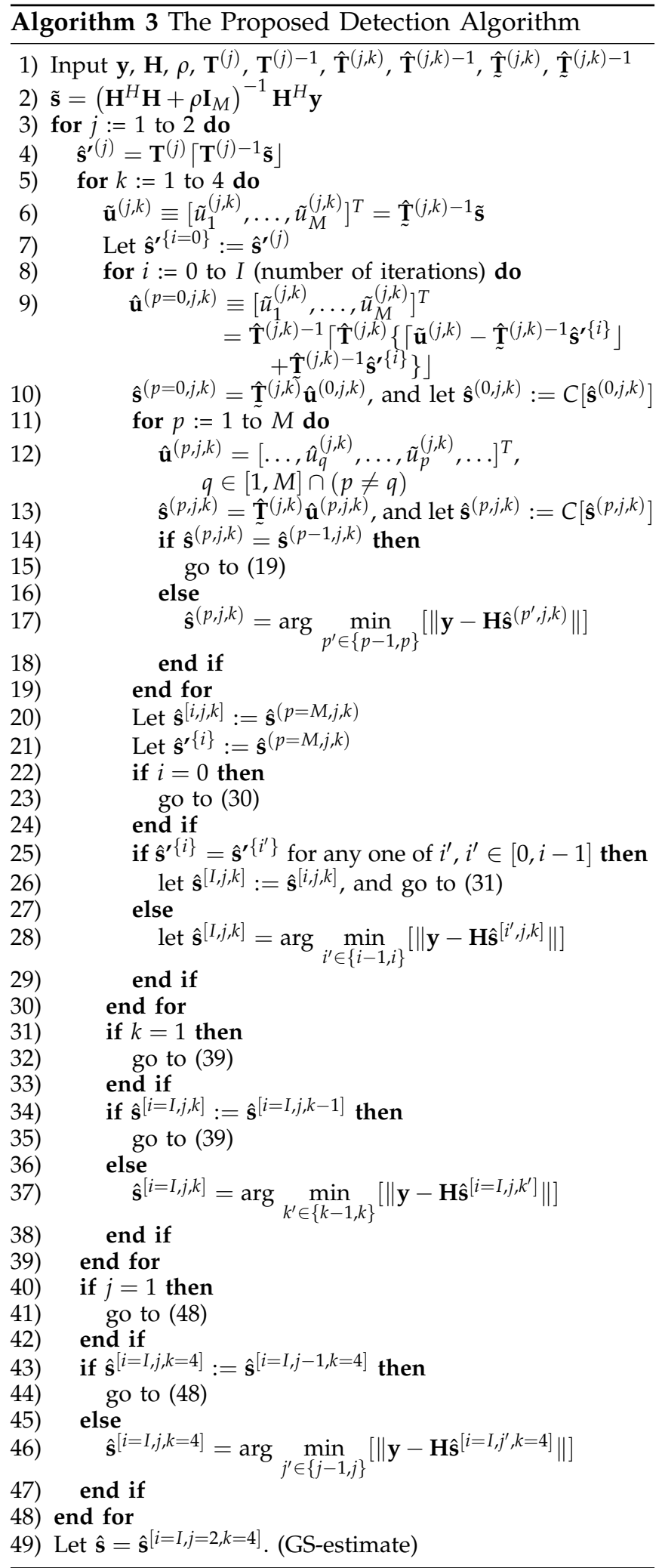

Table I summarizes the suitable values of $\delta$ and $I$ for the two modulation types.

\subsection{BER Performances}

Figures 5(a) and 5(b) show the BER characteristics versus $E_{b} / N_{0}$, respectively, for QPSK and 16QAM in the $4 \times 4$ MIMO system, with the same $\delta$ as shown in Table I. In each figure, BER curves with legends (1) and (2) are derived using the conventional and the proposed

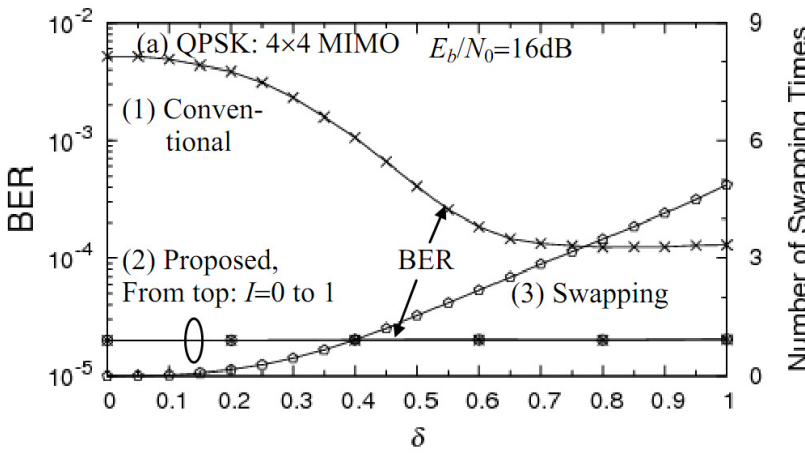

(a)

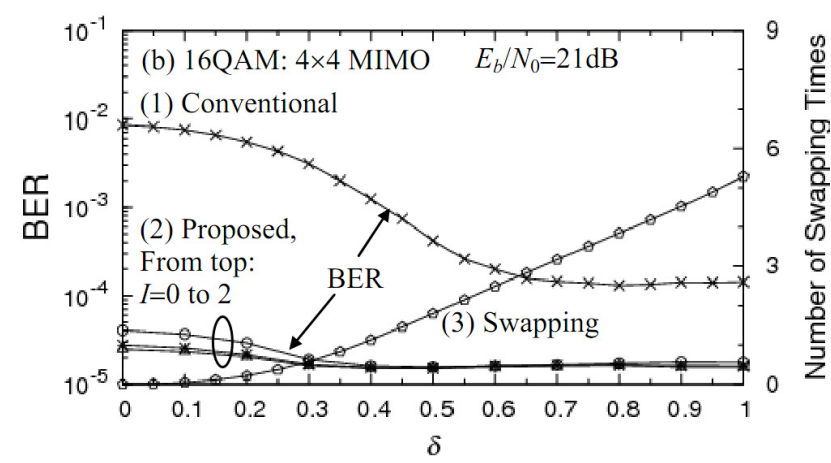

(b)

Figure 3. Characteristics of BERs and the number of swapping times versus $\delta$, in $4 \times 4$ MIMO. (a)- QPSK at $E_{b} / N_{0}=16 \mathrm{~dB}$, (b)- 16QAM at $E_{b} / N_{0}=21 \mathrm{~dB}$. Curves (1) and (2) are BERs for the conventional and the proposed detections, respectively. Curves (3) are the number of swapping times.

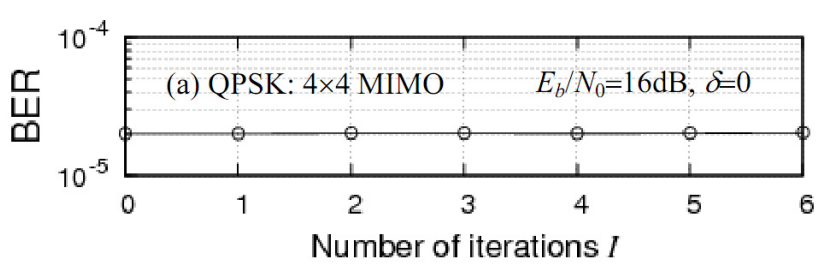

(a)

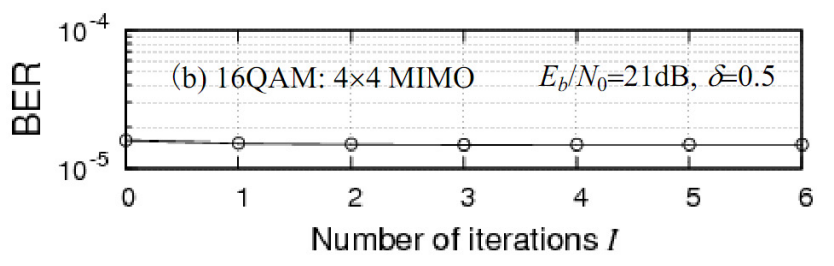

(b)

Figure 4. BER characteristics versus the number of iterations $I$ for the proposed detection, in $4 \times 4 \mathrm{MIMO}$; (a)- QPSK at $E_{b} / N_{0}=16 \mathrm{~dB}$ with $\delta=0,(b)-16 \mathrm{QAM}$ at $E_{b} / N_{0}=21 \mathrm{~dB}$ with $\delta=0.5$.

detections, respectively. BER curve (2) for QPSK is equivalent to the BER curves for the ML detection, same with BER curves for 16QAM. Hence we can obtain almost the same BER performance as that for the ML detection. As a consequence, the proposed detection dramatically improves the BER performance, which is almost equivalent to that using the ML detector. 
Table I

Suitable VAlues for $\delta$ AND $I$

\begin{tabular}{|l|c|c|}
\hline & QPSK & 16QAM \\
\hline Conventional detection & $\delta=0.75$ & $\delta=0.75$ \\
\hline Proposed detection: & $\delta=0$ & $\delta=0.5$ \\
$j \in\{1,2\}, k \in[1,4]$ & $I=0$ & $I=0$ \\
\hline
\end{tabular}

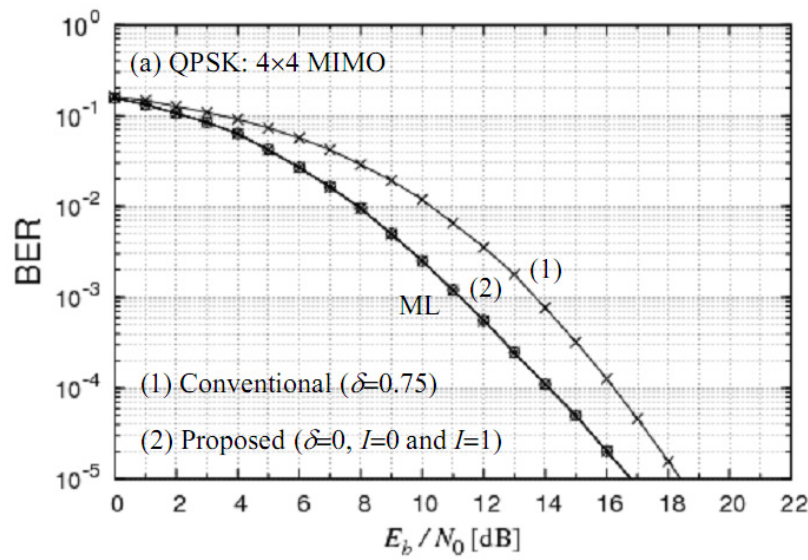

(a)

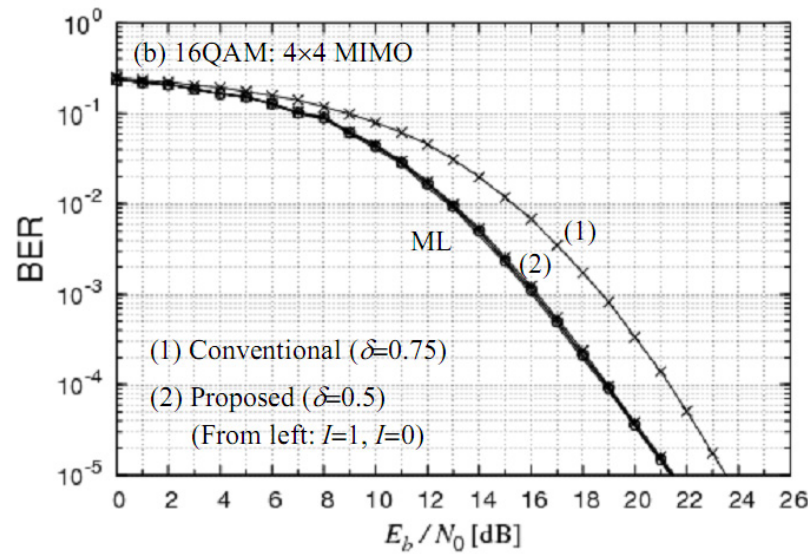

(b)

Figure 5. BER characteristics versus $E_{b} / N_{0}$, in $4 \times 4$ MIMO. (a)QPSK, (b)- 16QAM. BER curves (1) and (2) are derived using the conventional and the proposed detections, respectively. It should be noticed that in (a) there are two curves in legend (2): one is for $I=0$ and the other is for $I=1$. As can be seen, both curves are equivalent. And that in (b) there are two curves in legend (2): the left most curve dotted with circle is for $I=1$, and the right most curve dotted with " $\times$ " is for $I=0$. As can be seen, both curves are almost equivalent.

\subsection{Computational Complexity}

We count up the number of multiplications of two complex values in Algorithms 1, 2, and 3, and the number of those multiplications of Equations (5), (12) and (20), and their related calculations, since those multiplications dominantly contribute to the computational complexity.

In Algorithm 1, the number of calculations of $\hat{\mathbf{h}}_{q}^{H} \mathbf{h}_{p}$ in step (4) and of $\hat{\mathbf{h}}_{q}^{H} \hat{\mathbf{h}}_{p}$ in step (9) at $p$ with $q \in[1, p-1]$ is a total of $2(p-1)$. Hence, with weak reduction, the total number of those calculations over $p \in[2, M]$ is $\sum_{p=2}^{M} 2(p-1)=M(M-1)$. Since both $\hat{\mathbf{h}}_{p}$ and $\mathbf{h}_{p}$ have $2 M$ entries each, we have $2 M^{2}(M-1)$ multiplications for the above calculations. The number of divisions in both steps (4) and (9) is $M(M-1)$. For the squared norms $\left\|\hat{\mathbf{h}}_{q}\right\|^{2}$ in steps (4) and (9) at $p$ with $q \in[1, p-1]$, we need to calculate only $\left\|\hat{\mathbf{h}}_{p-1}\right\|^{2}$ at $p$, since the other $\left\|\hat{\mathbf{h}}_{q}\right\|^{2}$ for $q \in[1, p-2]$ have already been calculated before $p$ in the for-loop of $p$. Hence, with weak reduction, we have a total of $2 M \cdot M(M-1)$ multiplications for $\left\|\hat{\mathbf{h}}_{p-1}\right\|^{2}$ over $p \in[2, M]$. The number of calculations in both steps (5) and (10) at $p$ is a total of $3(p-1)$. With weak reduction, we have a total of $2 M \sum_{p=2}^{M} 3(p-1)=3 M^{2}(M-1)$ multiplications over $p \in[2, M]$.

If a column-swapping occurs at $p$, then $p$ goes back to $(p-1)$. The number of calculations of both $\hat{\mathbf{h}}_{q}^{H} \mathbf{h}_{p}$ in step (4) and $\hat{\mathbf{h}}_{q}^{H} \hat{\mathbf{h}}_{p}$ in step (9) at $p$ and the number of divisions of them by $\left\|\hat{\mathbf{h}}_{q}\right\|^{2}$ at $p$ are $2(p-1)$ each, and those at $(p-1)$ are $2(p-2)$ each. Hence we have $2 M$. $\{2(p-1)+2(p-2)\}=4 M(2 p-3)$ multiplications and $\{2(p-1)+2(p-2)\}=2(2 p-3)$ divisions due to the column-swapping at $p$. The number of multiplications in both steps (5) and (10) at both $p$ and $(p-1)$ is a total of $2 M \cdot\{3(p-1)+3(p-2)\}=6 M(2 p-3)$. Similarly, the number of multiplications for $\left\|\hat{\mathbf{h}}_{p-1}\right\|^{2}$ at $p$ and for $\left\|\hat{\mathbf{h}}_{p-2}\right\|^{2}$ at $(p-1)$ in both steps (4) and (9) is a total of $2 M \cdot 2=4 M$. Let $a$ be the average columnswapping times. Assuming that the column-swapping occurs uniformly with respect to $p$, the total number of calculations in Algorithm 1 is

$$
\begin{aligned}
A_{\mathrm{I}}(M, a) & =(M-1)\left(2 M^{2}+M+2 M+3 M^{2}\right) \\
& +\frac{a}{M-1} \sum_{p=2}^{M}\{(4 M+2+6 M)(2 p-3)+4 M\} \\
& +4 M(M-1+2 a) \\
& =M(M-1)(5 M+7)+2 a\left(5 M^{2}+2 M-1\right)
\end{aligned}
$$

on average. Here the term $4 M(M-1+2 a)$ is the number of multiplications for the squared norm of the right hand side of the inequality at step (12). Note that the left hand side $\left\|\hat{\mathbf{h}}_{p-1}\right\|^{2}$ in step (12) has already been calculated in step (4). Also note that according to computer simulation, the probability of the occurrence of the column-swapping at each $p, p \in[2,4]$, in Algorithm 1 for the $4 \times 4$ MIMO system is almost the same: the probabilities at $p=2,3$ and 4 are $31 \%, 40 \%$ and $29 \%$, respectively. Therefore, the actual number of multiplications in Algorithm 1 should be almost equal to $A_{\mathrm{I}}(M, a)$.

In Algorithm 2, the column vectors of $\mathbf{H}^{\prime}$ are weakly reduced. Hence, the total number of multiplications for $\hat{\mathbf{h}}_{q}^{H} \hat{\mathbf{h}}_{p}$ and that for $\left\|\hat{\mathbf{h}}_{q}\right\|^{2}$ in step (4) over $p \in[2, M]$ are $M^{2}(M-1)$ and $2 M(M-1)$, respectively. The number of divisions in step (4) is $M(M-1) / 2$. The total number of multiplications in step (5) is $2 M^{2}(M-1)$. As a result, the total number of multiplications in Algorithm 2 is

$$
A_{\mathrm{II}}(M)=(M-1)\left(M^{2}+2 M+\frac{1}{2} M+2 M^{2}\right) \text {. }
$$

To derive the inverse of an $M \times M$ matrix, we used the LU decomposition, which requires $4 M^{3} / 3$ multiplications [11]. Hence, the calculation of $\left(\mathbf{H}^{H} \mathbf{H}+\right.$ $\left.\rho \mathbf{I}_{M}\right)^{-1} \mathbf{H}^{H} \mathbf{y}(=\widetilde{\mathbf{s}})$ in (5) requires the number of multiplications of $S(M)=\frac{1}{2} M^{2}(M+1)+\frac{4}{3} M^{3}+2 M^{2}$. Both 
$\hat{\mathbf{s}}^{(j)}=\mathbf{T}^{(j)} \hat{\mathbf{v}}^{(j)}$ in $(20)$ and $\tilde{\mathbf{v}}^{(j)}=\mathbf{T}^{(j)-1} \tilde{\mathbf{s}}$ require the total number of multiplications of $T(M)=2 M^{2}+\frac{4}{3} M^{3}$ for each $j$. Both $\hat{\mathbf{T}}^{(j, k)}=\mathbf{T}^{(j)} \hat{\mathbf{T}}^{(j, k)}$ and $\tilde{\mathbf{u}}^{(j, k)}=\hat{\mathbf{T}}^{(j, k)-1} \tilde{\mathbf{s}}$ in (12) require the total number of multiplications of $U(M)=\frac{1}{2} M^{2}(M+1)+\left(\frac{4}{3} M^{3}+M^{2}\right)$ for each $j$ and $k$.

In the proposed detection algorithm of Algorithm 3, the equations in steps (14), (34) and (43) hold in high probabilities of more than $80 \%, 95 \%$ and $99 \%$ at a $\mathrm{BER} \approx 10^{-5}$, respectively. The equation in step (20) holds in high probabilities of more than $97 \%$ and $98 \%$ for $i=1$ and $i \geq 2$, respectively. Hence the steps (17), (28), (37) and (46) are seldom needed to calculate. This fact implies that they negligibly contribute to the computational complexity.

Since $\hat{\mathbf{T}}^{(j, k)-1}$ has already been derived, $\hat{\mathbf{T}}^{(j, k)-1} \hat{\mathbf{s}}^{(j)}$ in step ( $\tilde{9})$ requires $M^{2}(I+1)$ multiplications for each $j$ and $k$, and $i \in[0, I]$. Since both $\hat{\mathbf{T}}^{(j, k)}$ and $\hat{\mathbf{T}}^{(j, k)-1}$ are upper triangular matrices, the pre-multiplication by them in step (9) requires a total of $M(M+1)(I+1)$ multiplications for each $j$ and $k$, and $i \in[0, I]$. Similarly, $\hat{\mathbf{T}}^{(j, k)} \hat{\mathbf{u}}^{(0, j, k)}$ in step $(10)$ and $\hat{\mathbf{T}}^{(j, k)} \hat{\mathbf{u}}^{(p, j, k)}, p \in[1, M]$, in step (13) require $M^{2}(I+1)$ and $M \cdot M^{2}(I+1)=M^{3}(I+$ 1) multiplications, respectively, for each $j$ and $k$, and $i \in[0, I]$. As a result, for each $j$ and $k$, and $i \in[0, I]$, the number of multiplications in Algorithm 3 is summed up as

$$
\begin{aligned}
A_{\mathrm{III}}(M, I) & =\left\{2 M^{2}+M^{3}+M(M+1)\right\}(I+1) \\
& =M\left(M^{2}+3 M+1\right)(I+1) .
\end{aligned}
$$

It should be noted that all the calculations in steps (9), (10) and (13) are not done since the process skips out of the for-loop of $i$ at $i<I$ in step (25). This process decreases the iteration times. Hence the actual number of multiplications in Algorithm 3 is smaller than $A_{\text {III }}(M, I)$.

We first count up the number of multiplications for QPSK in the $4 \times 4$ MIMO system, where $M=4$. As we set $\delta=0.75$ for the conventional detection in Table I, the average swapping times are $a=3.7$ from Figure 3. The total number of multiplications for the conventional detection is in general expressed as $N_{\text {conv. }}(M, a) \equiv$ $A_{\mathrm{I}}(M, a)+S(M)+T(M)$. Substituting $M=4, a=3.7$ into the above, we have $N_{\text {conv. }}(M=4, a=3.7)=1242$.

For the proposed detection, we set $\delta=0$ and $I=0$ for QPSK in Table I. From Figure 3, the average swapping times are $a=0$. As we chose $j \in\{1,2\}$, the LLL algorithm is used twice. Hence the total number of multiplications in Algorithm 1 is $2 A_{\mathrm{I}}(M=4, a=0)$. We further reduce the columns of $\overline{\mathbf{H}}^{\prime(j, k)}$ for each $j$ and $k$ using Algorithm 2. Since $j \in\{1,2\}$ and $k \in[1,4]$, the total number of multiplications in Algorithm 2 is $8 A_{\text {II }}(M=4)$. The total number of multiplications in Algorithm 3 is $8 A_{\mathrm{III}}(M=4, I=0)$, where the skippingout process at step (25) in the for-loop of $i$ is not taken into account. The number of multiplications for (5), (12), (25), $\mathbf{T}^{(j)} \hat{\mathbf{T}}^{(j, k)}$ and $\mathbf{T}^{(j)-1} \tilde{\mathbf{s}}$ is a total of $S(M)+$ $2 T(M)+8 U(M)$. The total number of multiplications for the proposed detection is in general expressed as $N_{\text {prop. }}(M, a, I \mid k \in[1,4])=2 A_{\mathrm{I}}(M, a)+8 A_{\mathrm{II}}(M)+$
$8 A_{\text {III }}(M, I)+S(M)+2 T(M)+8 U(M)$. Substituting $M=4, a=3.7$ and $I=0$ into the above, we have $N_{\text {prop. }}(M=4, a=0, I=0 \mid k \in[1,4])=4490$. Hence we have $N_{\text {prop. }}(4,0,0 \mid k \in[1,4]) / N_{\text {conv. }}(4,3.7)=3.6$.

Next let us count up the number of multiplications for 16QAM. As we set $\delta=0.75$ for the conventional detection, the average swapping times are $a=3.7$ from Fig. 3. Hence the total number of multiplications is $N_{\text {conv. }}(M=4, a=3.7)=1242$.

For the proposed detection, we set $\delta=0.5$, and $I=0$ and $I=1$. Since the curve (2) with $I=0$ almost agrees with that with $I=1$, we chose the curve (2) with $I=0$. The average swapping times are $a=1.6$. In the same manner in the QPSK case, we got the total number of multiplications for the proposed detection as $N_{\text {prop. }}(M=4, a=1.6, I=0 \mid k \in[1,4])=$ 5047 for $j \in\{1,2\}$ and $k \in[1,4]$. Hence we have $N_{\text {prop. }}(4,1.6,0 \mid k \in[1,4]) / N_{\text {conv. }}(4,3.7)=4.0$.

As a result, we can obtain very good BER performances which are almost equivalent to those with the ML detection, if we allow around 3.6 through 4.0 times larger computational complexity, compared to the conventional LRA MMSE detection in the $4 \times 4$ MIMO system.

\section{Conclusions}

In this paper, we proposed a Gram-Schmidt based LRA MMSE list detector. First we forward- and backwardreduced the column vectors of the extended channel matrix using the LLL algorithm to create two reduced channel matrices. Those LLL-reduced column vectors are forward- and backward-reduced using the GS procedure to create the GS-reduced channel matrices, using which we obtained better reliable estimate of the transmitted signal.

The proposed detector dramatically improved the BER performances for QPSK and 16QAM in the $4 \times 4$ MIMO system. It achieved very good BER performances at the cost of a bit larger computational complexity. The BER curves are almost equivalent to those for the ML detector. This is because the GS procedure creates the column vectors of the reduced channel matrix to be mutually orthogonal. As a consequence, the proposed detector is worthy for applying to the $4 \times 4$ MIMO system.

\section{REFERENCES}

[1] T. D. Nguyen, X. N. Tran, and T. Fujino, "Layered error characteristics of lattice-reduction aided V-BLAST detectors," IEICE Transactions on Fundamentals of Electronics, Communications and Computer Sciences, vol. E89-A, no. 10, pp. 2535-2542, Oct. 2006.

[2] D. Wübben, R. Böhnke, V. Kühn, and K. D. Kammeryer, "Near-maximum-likelihood detection of MIMO systems using MMSE-based lattice reduction," in Proc. IEEE Int. Conference on Communications (ICC), vol. 2, Jun. 2004.

[3] X. Wang, Z. He, K. Niu, W. Wu, and X. Zhang, "An improved detection based on lattice reduction in MIMO systems," in Proc. IEEE Int. Symposium on Personal, Indoor and Mobile Radio Communications (PIMRC), Sep. 2006. 
[4] H. Yao and G. W. Wornel, "Lattice-reduction-aided detector for MIMO communication systems," in Proc. IEEE Global Telecommunications Conference (GLOBECOM), vol. 1, Nov. 2002, pp. 424-428.

[5] T. Fujino and T. Shimokawa, "Combined forward and backward lattice-reduction aided MMSE detection in MIMO systems," in Proc. IEEE Vehicular Technology Conference (VTC), Calgary, Canada, Sep. 2008.

[6] T. Fujino, S. Wakazono, and Y. Sasaki, "A Gram-Schmidt based lattice-reduction aided MMSE detection in MIMO systems," in Proc. IEEE Global Telecommunications Conference (GLOBECOM), Honolulu, USA, Dec. 2009.

[7] C. Windpassinger and R. F. H. Fischer, "Low-complexity near-maximum-likelihood detection and precoding for MIMO systems using lattice reduction," in Proc. IEEE Information Theory Workshop (ITW), France, France.

[8] K. L. Clarkson, W. Sweldons, and A. Zheng, "Fast multiple-antenna differential decoding," IEEE Transactions on Communications, vol. 49, no. 2, pp. 253-261, Feb. 2001.

[9] A. K. Lenstra, H. W. Lenstra, and L. Lovász, "Factoring polynomials with rational coefficients," Mathematische Annalen, no. 261, pp. 515-534, 1982.

[10] H. Hassibi, "An efficient square-root algorithm for BLAST," in Proc. IEEE Int. Conference on Acoustic, Speech and Signal Processing, Istanbul, Turkey, 2000, pp. 5-9.

[11] A. Kaw, Introduction to matrix algebra, ser. ISBN: 978-0615-25126-4. Autarkaw.com, 2008.

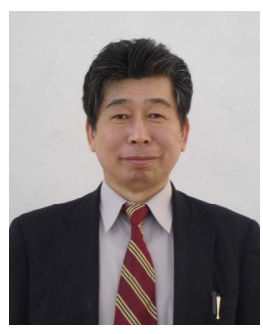

Tadashi Fujino was born in Osaka, Japan on 15 July, 1945. He received B.E. and M.E. degrees in electrical engineering and Dr. Eng. degree in communication engineering from Osaka University, Osaka, Japan, in 1968, 1970, and 1985, respectively.

Since April 2011, he has been Professor Emeritus of The University of ElectroCommunications (UEC), Tokyo, Japan. From April 2000 to March 2011, he was an ordinary professor in wireless communications at Department of Information and Communication Engineering and Informatics, Graduate School of Informatics and Engineering, The UEC, Tokyo, Japan. Before then, he had been with Mitsubishi Electric Corporation, Tokyo, Japan since 1970, where he devoted in R\&D in the wireless communications area such as digital satellite communications and digital land mobile communications. His major works include the feasibility study and the hardware development of the $120 \mathrm{Mbps}$ trellis coded (TC) 8-PSK burst modem for TDMA system. This work was done at INTELSAT's request, and is the first development in the world. In the above feasibility study and the hardware development, he made two innovative inventions: one is the "pipe-lined Viterbi decoder," which makes possible for TC modems to operate in very high bit rates of hundreds Mbps, and the other is the clock aided carrier recovery system for the coherent detection of M-PSK modulated signal, which generates very little pattern noise. After designing and manufacturing the hardware of the TC burst modem using these inventions, he made field test, which was successfully done. By his two inventions and the field test results, it is verified that the high bit rate TC burst modem should be feasible to design. His current interests include the signal detection in MIMO systems such as lattice-reduction aided detection. He wrote a single authored book "Digital mobile communication," and three coauthored books. He received Meritorious Award from the ARIB (The Associate of Radio Industries and Businesses of Japan) of MPT of Japan, in 1997. He is also a Visiting Professor at Bac-Ha International University, Hanoi, Vietnam.

Dr. Fujino is a Fellow of IEEE and a member of IEICE. 